\title{
When should placebo surgery as a control in clinical trials be carried out?
}

\section{Placebo surgery - often maligned as 'sham surgery' - is a tough sell to patients and to many clinicians. But could surgical research benefit from increased use of placebo control groups?}

AJT George Chair, National Research Ethics

Advisors' Panel, Health Research Authority; Deputy

Vice-Chancellor, Brunel University London

C Collett Ethics Guidance and Strategy

Manager, Health Research Authority

AJ Carr Head, Nuffield Department of

Orthopaedics, Rheumatology and Musculoskeletal

Sciences, University of Oxford

S Holm National Research Ethics Advisors

Panel, Health Research Authority; Professor

of Bioethics, University of Manchester

C Bale Head of Research Communications

and Engagement, Parkinson's UK

S Burton Head of Standards and

Ethics, General Medical Council

M Campbell Director, Health Services

Research Unit, University of Aberdeen

\author{
A Coles Professor of Neuroimmunology, \\ University of Cambridge \\ G Gottlieb Policy Officer, RCS \\ K Muir Professor of Clinical Imaging, University of Glasgow \\ S Parroy Lay Vice-Chair, Patient and Lay Group, RCS \\ $J$ Price Head, Centre for the Cellular Basis of \\ Behaviour, Institute of Psychiatry, Psychology \\ and Neuroscience, King's College London \\ ASC Rice Professor of Pain Research, \\ Imperial College London \\ J Sinden Chief Scientific Officer, ReNeuron \\ C Stephenson Research Support \\ Network Manager, Parkinson's UK \\ K Wartolowska Research Fellow, Nuffield \\ Department of Orthopaedics, Rheumatology and \\ Musculoskeletal Sciences, University of Oxford \\ H Whittall Director, Nuffield Council on Bioethics
}


$\mathbf{T}$ he efficacy of novel surgical therapies needs rigorous evaluation in clinical trials. These studies need to be appropriately designed and controlled. In clinical trials of drugs, it is common to use placebo controlled groups to account for the psychological placebo effect and to ensure that the patient (and often the researcher) is unaware of whether he or she is receiving the experimental treatment. However, relatively few surgical trials involve placebo components, often unhelpfully termed 'sham surgery'.

Following a workshop on the use of placebo controls in surgery, ${ }^{1}$ we argue that placebo has an important role in surgical research, not only to control for the psychological placebo effect but also (when surgical approaches are used) to deliver the putative therapeutic agent so as to determine whether any therapeutic benefit is as a result of the agent or the surgery. We highlight guidelines on the use of placebo controls in surgical research and argue that placebo interventions should be used more often to control surgical studies, as this will often produce the best research evidence.

In order to deliver the best clinical care to patients, treatments need to be based on evidence gained through clinical research. This applies to surgical interventions just as much as to pharmaceutical treatments. Nevertheless, in many cases new surgical techniques or approaches are introduced with little in the way of clinical trials, and the quality and quantity of randomised trials of surgical techniques is limited.' This means that we are often unsure of whether surgical treatment is effective and, indeed (as has been the case with pharmaceutical trials), ${ }^{2}$ studies that have adopted a scientifically rigorous design have shown that common surgical treat-

'A joint workshop of the Nuffield Council on Bioethics and the Health Research Authority was organised to consider a recommendation of the Nuffield report Novel Neurotechnologies: Intervening in the Brain (www.nuffieldbioethics.org/neurotechnology). The group met on 1 May 2015. This publication is a consequence of the meeting but does not necessarily represent the opinion of the Health Research Authority, the Nuffield Council or the employers of participants at the workshop. ments are often not effective. For example, a rigorously controlled trial failed to demonstrate any advantage for arthroscopic lavage or debridement (both commonly performed techniques) over a placebo procedure in osteoarthritis of the knee. ${ }^{3}$

For the purposes of this article, we define 'surgery' in its broadest context, to include not only its conventional usage but also invasive (usually 'needle-based' percutaneous) procedures. These are increasingly performed by suitably trained non-surgical specialists in areas such as pain medicine and radiology. We would also extend this usage to the implantation of neuromodulator devices and devices for therapeutic substance delivery.

The gold standard of clinical evidence is usually considered to be the randomised controlled trial. This can provide the highest-quality evidence for the efficacy (or otherwise) of a new form of treatment. Meta-analysis of a number of studies can further increase confidence in the outcome. Randomised controlled trials, especially those on pharmacological interventions, involve the use of a group selected randomly to receive a placebo intervention.

The use of placebo is important because of the multiple psychological and neurobiological effects that placebo treatment can have. ${ }^{6.7}$ It can also ensure that the evaluation of an effect is unbiased (by masking both the patient and, often, the researcher as to whether the subject is in the treatment group or the control group). Placebo controls allow for the 'true' effect of treatment to be separated from the placebo effects. The magnitude of the response to placebo is context and suggestion dependent; for example, in studies using medical devices, the placebo effect may be particularly strong. ${ }^{8}$ It is important to recognise that placebo and nocebo effects can contribute not only to the effect observed in the placebo group but also to the efficacy of active intervention.

Placebo controlled groups can be used to estimate the magnitude of placebo responses. In such cases, their use only applies where

\section{Box 1 Definitions $s^{4,5}$}

Placebo is a dummy treatment such as inert medication or sham surgery, which can vary from a minor procedure through to a full surgical procedure with the critical element under investigation omitted.

Placebo response is an effect observed in an individual that results from the expectation of effectiveness of the therapeutic intervention. 'Placebo response' is sometimes erroneously used to describe any type of improvement observed in a placebo assigned group in a clinical trial, even if that improvement may in fact relate to statistical factors (sampling bias, regression to the mean) or the natural history of the condition.

Placebo effect is an observable difference between groups that is attributable to the efficacy of the placebo

Nocebo effect describes a negative or adverse response following administration of an inert treatment. Nocebo effects mirror the placebo effects but are an independent phenomenon.

placebo effects might have an impact on outcome. This is particularly relevant to areas that are reliant on patient-reported outcomes (eg where the intervention is intended to deliver pain relief). When the assessment of interventions is very objective (eg the outcome of cardiac transplantation), then it will not be necessary to include a placebo control group to account for the psychological placebo effect.

However, placebo can also be important in the design of studies for reasons other than the psychological or neurobiological effect of the intervention. Placebo studies are also effective in helping determine the mechanism of action of the therapy. For example, in cases where the surgery or implanted device is used to deliver a putative therapeutic agent, a placebo-controlled group may be used in which an inactive substance is delivered so as to determine whether any therapeutic effect is due to the surgical intervention (eg as a result of an inflammatory response) or the supposed active substance. In such cases, the use of a placebo might be warranted even when the measured outcome is objective.

In a systematic review from 2014, only 53 surgical studies in the period up to Novem- 
ber 2013 were identified as being described as placebo controlled. ${ }^{9}$ In half of these studies, the results provided evidence against continued use of the investigated surgical procedures because the surgery was not more effective than placebo. The fact that placebo-controlled trials of surgery are rarely conducted means that ineffective surgical treatment may continue unchallenged.

The use of placebo surgical interventions is sometimes referred to as 'sham surgery' but the term 'placebo surgery' is preferred to the pejorative and misleading term 'sham'. Such interventions can vary from a minor procedure such as making a small incision in the skin (so that a patient does not know whether he or she has had the investigative procedure) to a full surgical procedure with the critical surgical element under investigation omitted. For example, in a trial of neural stem cell therapies, a control group undergoing surgery where saline solution alone is injected (instead of stem cells in suspension) would be required to accurately control for not just the placebo effect but also the biological effect of injecting material into the brain.

A scientifically rigorous approach of this kind has been adopted in a current study of arthroscopic acromioplasty for shoulder pain, where patients are randomised to receive the acromioplasty or, as a placebo, arthroscopy alone (with a third group having active monitoring). ${ }^{10}$ Other examples of placebo surgery involve the drilling of burr holes in the skull of patients who were randomised to not receive stereotactic delivery of fetal cells for Parkinson's disease." This had the dual purpose of not allowing recipients to know whether they had received the cells (providing a placebo control) and also of masking the research team as to which group the patients were belonged, thereby allowing more objective assessment of the outcome. The drilling of burr holes does not address the potential biological effects of brain lesioning entailed in stereotaxic injection, which may have an independent biological effect on motor systems.

Scenario 1 Masking + placebo effect

\section{Example}

The study is of a surgical technique that results in a small incision. The placebo intervention might involve a small surgical incision to produce a wound that is similar to that seen in the investigational treatment group.

\section{Considerations}

- The placebo intervention should be chosen to be the least invasive and risky that can achieve effective masking and control for non-specific effects of therapy.

- Careful thought needs to be given as to whether the placebo intervention is an effective control or whether there will be differences in things such as procedural duration that would effectively unmask the study.

- The risk-benefit analysis should be similar to that for pharmaceutical studies.

Scenario 2 Intervention may have a non-specific effect

\section{Example}

Stem cell treatment in which the mechanical means by which the cells are injected into the tissue lor the fluid the tissue is in) may have an effect that is independent of the cells (or an unexpected 'non-specific' response to the cells). The placebo intervention in this case would involve injection of the fluid, without cells, into the tissue. This controls both for the placebo effect and the non-specific biological effect of the intervention as well as masking the participant and researchers as to which group the patient is in.

\section{Considerations}

- Is there an alternative study design (such as cross-over or double dummy) that does not involve placebo intervention and can answer the research question?

- Is there good evidence that there might be a non-specific effect of treatment (eg from an animal model)?

- The placebo intervention should be chosen to be the least invasive and risky that can achieve effective masking and control for non-specific effects of therapy.

The risk-benefit analysis should be similar to that for pharmaceutical studies.

Scenario 3 Long-term intervention

\section{Example}

Implantation of an electrode assembly for deep brain stimulation. The placebo intervention may involve the implantation of a device that is not activated or that is programmed to switch randomly between active and inactive modes. This could mask the participant and researcher as to which group the patient is in and also control for non-specific effects of surgery. This could also allow the device to be activated later.

\section{Considerations}

- Is there an alternative study design that can answer the research question? (In this case, a crossover study might be considered.)

- Is there good evidence that there might be a non-specific effect of treatment?

- The placebo intervention should be chosen to be the least invasive and risky that can achieve effective masking and control for non-specific effects of therapy.

- The risk-benefit analysis should be similar to that for pharmaceutical studies although possibly more complex than that in the other scenarios.

- There is a specific problem relating to the frequently made promise that if the treatment is found to be effective, participants in the control group will receive it later (ie have the device turned on). Participants may interpret this not as 'if..., then...' but as 'not now but later'. Participants need to be made aware that should the intervention not be shown to be effective, then (if they are in the control group) they will not get it.

Various ethical arguments have been raised against the use of placebo controls in surgical research. It has been suggested that the use of placebo surgical controls involves deception of the participant. However, where a participant in research is fully informed as to the possibility of being randomised to receive either the placebo treatment or the investigational intervention, this 'deception' is no more problematic than placebo-controlled drug trials. Similarly, in psychological research, the use of deception (followed by 
debriefing) is widely used and considered to be acceptable, provided it is justified by the social and scientific value of the research, and an appropriate risk management and harm alleviation strategy is in place. ${ }^{12}$

It has also been suggested that because placebo surgery is more invasive than placebo medicines (and therefore potentially involves greater physical harm), it is much more difficult to justify its use in research. 13,14 On the other hand, ethical review ensures that any harm involved is minimised, reasonable and justified by the potential individual and societal benefits that might accrue. Such review also ensures that the potential participants are given the information needed to make an informed decision about their participation and the risks and benefits involved. Each research project needs to be considered on its own merits and placebo surgery is no different in that respect to other trials that involve placebo interventions. This ethical point is also countered by the population level harm incurred by the perpetuation in clinical practice of interventions that are found to be ineffective once compared with placebo.

There is also a fear that placebo trials will involve patients being denied treatment that is known to be effective. However, whether used in surgical or any other research, placebo controls should not normally replace any standard treatment that is known to be effective. Research should only be conducted where there is equipoise (ie genuine uncertainty regarding the relative therapeutic merits of each of the interventions involved in the research). As in drug trials, it is not normally acceptable for patients to be randomised to receive either the experimental treatment or placebo when there is a proven known effective therapy. This is to ensure that patients are not denied an effective therapy and also that the experimental drug is compared against standard current practice.

It is often possible to mitigate some of these ethical considerations by careful trial design. A study in which a device for brain stimulation is implanted could lend itself to a cross-over design in which the device is 'turned off' (placebo) or delivers the stimulation.

Despite this, the use of placebo surgical interventions can often evoke strong opinions both for and against their use. In one study exploring the feasibility and acceptability of placebo controls for arthroscopic lavage among clinicians, patients and chairs of research ethics committees, there was widespread acceptance that the use of placebo was necessary if scientific rigour was not to be compromised. ${ }^{15}$ Nevertheless, there was disagreement over how 'risky' (and therefore how ethically 'acceptable') the

\section{While the use of placebo controls may have more widespread acceptance among surgeons and researchers, such controls may not always be acceptable to patients}

proposed placebo was perceived to be. For example, there was a difference of opinion over whether patients in the placebo arm should receive general anaesthetic. Some believed that the use of placebo could not be justified under any circumstances.

In response to evidence that arthroscopic washout of the degenerate knee is no more effective than placebo surgery, ${ }^{16}$ leaders of the arthroscopic surgery community have asserted that patients who participate in placebo-controlled trials 'may not be of entirely sound mind' and that 'ethically, sham surgery is a questionable research method, which may be harmful'. In a study of surgeons, the vast majority (96\%) believed that it was acceptable to use placebo surgery, especially in clinical research (46\%). ${ }^{18}$ Only $4 \%$ believed it should always be prohibited, citing concerns over the element of deception of the patient and the risk to patient-surgeon trust.

Further data from a study that randomised patients to meniscectomy or placebo surgery showed that 102 of the 190 eligible patients declined to participate. ${ }^{19}$ Of these, almost a third said it was because they did not want a placebo operation. A qualitative study of the perspectives of patients with Parkinson's disease demonstrated that when presented with a hypothetical research proposal, a small majority of people were sympathetic to the concept of placebo surgery. ${ }^{20}$ However, a large majority stated that they would not want to receive placebo surgery as part of a research study, indicating that most patients do not consider that the experimental treatment is in equipoise with the placebo treatment.

These data suggest that while the use of placebo controls may have more widespread acceptance among surgeons and researchers, such controls may not always be acceptable to patients, thereby making their use in surgical research difficult. Patients' wishes may be dependent on the language used when conveying information. For example, they may be less likely to accept 'placebo treatment' if offered an alternative of 'surgical treatment' than if an 'experimental surgical operation' is the alternative being offered.

Under what situations might it be acceptable to use placebo surgery (and when might it not be)? Where there is no likelihood of a placebo effect (eg when the outcome of the surgery is objectively quantifiable and not reliant on patient or researcher reported outcomes such as pain relief), there may not be a need for placebo and a 'non-treatment' 
arm may provide sufficient evidence as to the effectiveness of the intervention. The exception to this would be when a placebo group is required to determine which part of a therapeutic intervention is effective (eg to differentiate between the biological effect of surgically delivering a substance and the effect of the substance itself). Where a placebo and/or biological effect is likely, many of the considerations that would need to be taken into account would be those that are common to all clinical research, such as:

- The research question being addressed should be sufficiently important.

- The researcher should be uncertain about the effectiveness of the treatment.

- The study design should ensure that patients are not given an ineffective placebo in place of a treatment that is known to be effective.

- The study design should be appropriate and sufficiently robust to answer the research question.

- The potential risk and burden to the research participant should be considered and minimised, taking into account the significance of the study, the potential benefit to the participant and the effectiveness of current treatment. (It is unlikely that total substitution of a current effective treatment with placebo would be acceptable.)

- The information given to the potential participant should be honest and clear so that he or she can make an informed decision as to whether to take part.

These are all factors that researchers and research ethics committees are used to taking into account. However, there will be other issues relating to the use of surgical placebos that may need to be considered. If the placebo treatment is associated with significant risk, then it may not be ethical to use it unless the possible individual and societal benefits far outweigh this. For example, it is inconceivable that it would ever be ethical to subject a patient to open heart surgery or amputation as part of a placebo control. Some forms of placebo may prevent future treatment (eg by forming scar tissue) and therefore raise specific issues. Many of these issues are discussed in useful guidelines developed by the American Medical Association. ${ }^{21}$

Based on the points raised in this article, we believe that placebo interventions should be used more frequently to control surgical studies as this will often produce the best research evidence. Every study should be considered on its merits, using standard approaches that are well developed for drug studies involving placebo. We have outlined three scenarios to give examples of the factors that should be considered.

\section{References}

1. McCulloch P, Taylor I, Sasako M et al. Randomised trials in surgery: problems and possible solutions. BMJ 2002; 324: 1,448-1,451.

2. Roberts I, Yates D, Sandercock P et al. Effect of intravenous corticosteroids on death within 14 days in 10,008 adults with clinically significant head injury (MRC CRASH trial): randomised placebo-controlled trial Lancet 2004; 364: 1,321-1,328.

3. Moseley JB, O'Malley K, Petersen NJ et al. A controlled trial of arthroscopic surgery for osteoarthritis of the knee. N Engl J Med 2002; 347: 81-88

4. Wager TD, Fields HL. Placebo Analgesia. In: McMahon SB, Koltzenburg M, Tracey I, Turk DC. Textbook of Pain. 6th edn. Philadelphia: Elsevier; 2013.

5. Vase L, Amanzio M. Price DD. Nocebo vs placebo: the challenges of trial design in analgesia research. Clin Pharmacol Ther 2015; 97: 143-150.

6. Price DD, Finniss DG, Benedetti F. A comprehensive review of the placebo effect: recent advances and current thought. Annu Rev Psychol 2004; 59: 565-590.

7. Benedetti F. Mechanisms of placebo and placeborelated effects across diseases and treatments. Ann Rev Pharmacol Toxicol 2008; 48: 33-60.

8. Kaptchuk TJ, Goldman P. Stone DA, Stason WB. Do medical devices have enhanced placebo effects? J Clin Epidemiol 2000; 53: 786-792.

9. Wartolowska K, Judge A, Hopewell S et al. Use of placebo controls in the evaluation of surgery: systematic review. BMJ 2014; 348: g3253.

10. Beard D, Rees J, Rombach I et al. The CSAW Study (Can Shoulder Arthroscopy Work?) - a placebocontrolled surgical intervention trial assessing the clinical and cost effectiveness of arthroscopic subacromial decompression for shoulder pain: study protocol for a randomised controlled trial. Trials 2015; 16: 210
11. Freed CR, Greene PE, Breeze RE et al. Transplantation of embryonic dopamine neurons for severe Parkinson's disease. N Engl J Med 2001; 344: 710-719.

12. British Psychological Society. Code of Human Research Ethics. Leicester: BPS; 2014.

13. Macklin R. The ethical problems with sham surgery in clinical research. N Engl J Med 1999; 341: 992-996.

14. London AJ, Kadane JB. Placebos that harm: sham surgery controls in clinical trials. Stat Methods Med Res 2002; 11: 413-427.

15. Campbell MK, Entwistle VA, Cuthbertson BH et al. Developing a placebo-controlled trial in surgery: issues of design, acceptability and feasibility. Trials 2011; 12: 50

16. Sihvonen R, Paavola M, Malmivaara A et al. Arthroscopic partial meniscectomy versus sham surgery for a degenerative meniscal tear. N Engl J Med 2013; 369: 2,515-2,524.

17. Carr A. Arthroscopic surgery for degenerative knee. BMJ 2015; 350: h2983

18. Wartolowska K, Beard DJ, Carr AJ. Attitudes and beliefs about placebo surgery among orthopedic shoulder surgeons in the United Kingdom. PLoS One 2014; 9: e91699.

19. Hare KB, Lohmander LS, Roos EM. The challenge of recruiting patients into a placebo-controlled surgical trial. Trials 2014; 15: 167.

20. Swift TL. Sham surgery trial controls: perspectives of patients and their relatives. J Empir Res Hum Res Ethics 2012; 7: 15-28.

21. Tenery R, Rakatansky H, Riddick FA et al. Surgical 'placebo' controls. Ann Surg 2002; 235: 303-307.

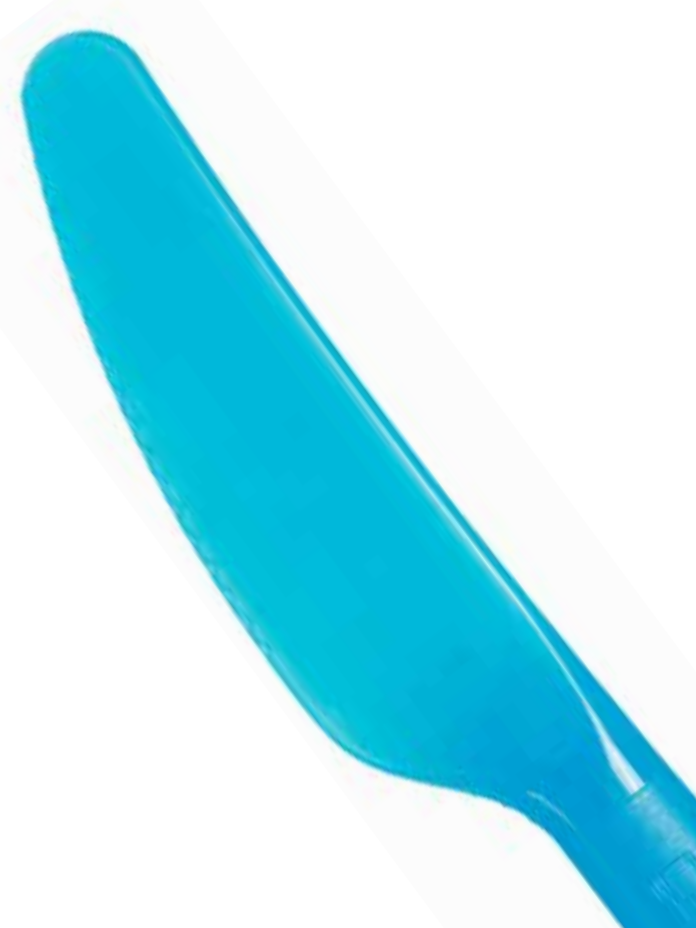

